\title{
Estudo analítico da interdisciplinaridade na composição dos membros dos Comitês de Ética em Pesquisa no Brasil\#
}

\section{Analytical study of interdisciplinariity regarding Research Ethics Committees membership in Brazil}

Rita de Cássia da Costa*

Fabiano Maluf**

\begin{abstract}
RESUMO: Criados a partir da Resolução n. 196/96, do Conselho Nacional de Saúde (CNS), os Comitês de Ética em Pesquisa (CEP) são parceiros no processo educativo de construção de uma cultura bioética para a condução de pesquisas que envolvem seres humanos. A composição dos membros de CEP é ratificada com a publicação da Resolução n. 466/12 do CNS, cujo colegiado deve ser composto por ambos os gêneros, multidisciplinar e multiprofissional, e pelo menos um representante de usuário. O presente trabalho teve como objetivo analisar o perfil da área de formação acadêmica dos membros que compóem os CEP no Brasil. Trata-se de um estudo documental, descritivo, retrospectivo e de abordagem quantitativa e foi baseado nos relatórios anuais e no banco de dados da Comissão Nacional de Ética em Pesquisa (CONEP), realizada no período compreendido entre 31 de agosto a 31 de Setembro de 2010. Em 2010 foram encontrados 602 Comitês registrados na CONEP, com 9.075 membros atuantes e uma média de 16 membros por comitê, distribuídos em diferentes áreas do conhecimento, a saber: Ciências da Saúde (54\%), Ciências Humanas (13\%), Ciências Sociais (11\%), Ciências Biológicas (5\%), Ciências Exatas (4\%), Engenharias (2\%), Agrárias (1\%), Linguística (1\%) e Outros (9\%). A multidisciplinaridade é respeitada na composição dos membros dos CEP com o predomínio das Ciências da Saúde e crescimento considerável de categorias como a Fisioterapia, a Fonoaudiologia e a Psicologia e, paulatinamente, a adesão progressiva de áreas anteriormente inexistentes ou escassas como Educação, Direito, Sociologia e Antropologia, o que permite inferir o interesse e o alcance que a ética em pesquisa tem despertado nessas áreas. Com o funcionamento regular dos Comitês infere-se o aumento anual dos projetos avaliados, o que reflete a estruturação do Sistema CEP-CONEP com o incremento real das atividades de pesquisa no país.
\end{abstract}

PALAVRAS-CHAVE: Ética em Pesquisa. Pesquisa Interdisciplinar. Bioética.

ABSTRACT: Ethics Committees (ECs) were established by Resolution 196/96 of National Health Council (NHC), and have a pivotal role in the process of building a bioethical culture in conducting all research involving human beings. ECs' membership was consolidated by Resolution $466 / 12$ of NHC, which states that the board should be composed by members from both genders, and must be multidisciplinary and multi-professional, in addition to having at least a users' representative. This article aims to analyze the profile of members of ECs according to their academic background in Brazil. This is a documentary study and a descriptive, retrospective and quantitative approach. It was performed based on the annual report and database of CONEP for the period from 31st August to 31st September 2010. In 2010, there were 602 registered committees in CONEP database, 9,075 active members and an average of 16 members per committee, distributed in different areas of knowledge, namely: Health Science (54\%), Humanities (13\%), Social Science (11\%), Life Sciences (5\%), Exact Science (4\%), Engineering (2\%), Agriculture (1\%), Linguistics (1\%) and others $(9 \%)$. Multidisciplinarity is sustained in the composition of ECs membership, in which the major academic area is Health Science. However there is a considerable growing of categories such as physiotherapy, speech therapy and psychology, and a gradual and progressive adherence of areas previously absent or scarce, such as Education, Law, Sociology and Anthropology, which allows us to infer that these areas have started taking interest in and reaching out for ethics in research. As the system for operational in ECs raises, there is an enhancement in studies analyzed, which reflects the well structured CEP-CONEP system, and the increasing of research activities in the country.

KEYWORDS: Ethics, Research. Interdisciplinarity Research. Bioethics.

DOI: $10.15343 / 1981-8254.20140801053060$

\# Baseado na monografia apresentada em 2011 no XII Curso de Especialização em Bioética realizado pela Cátedra Unesco de Bioética da Universidade de Brasília: “Costa RC. Estudo analítico da interdisciplinaridade na composição dos membros dos Comitês de Ética em Pesquisa [monografia]. Brasília: Faculdade de Ciências da Saúde, Universidade de Brasília; 2010. 40 p."

* Analista de Sistemas. Técnica da Comissão Nacional de Ética em Pesquisa - CONEP/CNS/MS. Especialista em Direito Público. Especialista em Bioética pela Universidade de Brasília - UnB. E-mail: rita.costa@saude.gov.br

** Cirurgião dentista. Especialista em Bioética. Doutorando em Ciências da Saúde com linha de pesquisa em Bioética pela Universidade de Brasília - UnB.

Os autores declaram não haver conflitos de interesse. 


\section{INTRODUĈ̣̃O}

As discussóes acerca das moralidades das pesquisas com seres humanos remontam à antiguidade grega e romana, passando pelos escritos de Maimônides e Bacon. De acordo com Werner e Campos Velho ${ }^{1}$, nos séculos XVIII e XIX, as pesquisas eram basicamente terapêuticas e eram realizadas em pequena escala. Em 1830, se percebeu a necessidade de introduzir parâmetros éticos nas pesquisas. O médico francês Claude Bernard, em um artigo sobre o estudo da Medicina, afirmou que somente seria válido realizar experimentos com humanos caso esses pudessem trazer algum benefício ${ }^{1}$.

Segundo Freitas e Novaes ${ }^{2}$, atualmente a regulamentação da pesquisa envolvendo seres humanos é parte das políticas públicas e práticas sociais nos países regidos por sistemas democráticos e visa ampliar e garantir os direitos dos cidadãos, no caso, especificamente, dos participantes de pesquisa.

Em anos recentes, tem sido estudada a influência que o perfil dos membros de Comitês de Ética em Pesquisa (CEP) exerce sobre suas práticas e deliberações cotidianas, devido à aplicação de referenciais éticos e regulamentações que ocorrem por meio de decisões em que o contexto social deve ser considerado.

O presente artigo resultou da análise de dados levantados em setembro de 2010, na Comissão Nacional de Ética em Pesquisa (CONEP). Foram consultados os relatórios anuais e o sistema interno de informação (Sistema Gestão) do órgão, ou seja, a análise contempla quatorze anos de atividade do Sistema CEP-CONEP. Assim, este estudo constitui subsídio para se fazer uma abordagem do quadro de evolução e organização dos CEP, sua importância ética e histórica, bem como, poder demonstrar o perfil acadêmico dos membros dos comitês de ética que compõem o Sistema.

\section{REVISÃO DA LITERATURA}

Historicamente, os Comitês de Ética em Pesquisa nascem como resposta às implicações morais do desenvolvimento técnico-científico na área biomédica, depois que foi desvendado que é possível, em nome da pesquisa e do progresso do conhecimento, cometer crimes hediondos contra a humanidade e contra os mais elementares direitos do cidadão ${ }^{3}$.

De acordo com Francisconi e Goldim ${ }^{4}$, os CEP não têm uma função estritamente cartorial e burocrática; desempenham um papel consultivo e educativo e servem como espaços para discussão e reflexão ética, com o intuito de salvaguardar os interesses da sociedade como um todo e dos indivíduos em particular. Ao aprovar um protocolo de pesquisa, o CEP passa a ser corresponsável pela parte ética do projeto.

Além disso, a CONEP tem como preocupação constante a transparência de suas açôes no cumprimento das atribuições ratificadas pela Resolução n. 466/12 recentemente publicada. Esse princípio é percebido na apresentação periódica de planos de trabalho e nos relatórios das atividades, assim como a implantação da Plataforma Brasil. A partir de 1997, as instituiçôes começaram a apresentar propostas de criação de comitês de ética locais, após análise prévia realizada pela CONEP e passaram a receber a aprovação e registro ou recomendação para adequação às normas.

Segundo Massarollo, et $\mathrm{al}^{6}$, os abusos e deslizes éticos não serão inteiramente evitados por diretrizes ou documentos. O efetivo controle ético envolve, principalmente, a sensibilização dos pesquisadores relativa à ética na pesquisa. A exigência de uma conduta ética pode indignar as pessoas, por parecer que é evidente demais e, portanto, desnecessária a colocação dessa questão. Entretanto, o fato é que as considerações de natureza ética nem sempre recebem a atenção apropriada.

De acordo com Freitas ${ }^{7}$, os CEP colocam outro olhar nos projetos de pesquisa fazendo com que os pesquisadores e patrocinadores não sejam os únicos a apreciarem se os seus projetos estão em conformidade com as diretrizes estabelecidas. As resistências, quando existem, originam-se, em especial, daqueles que acreditam que a competência ética sempre acompanha a competência técnica. 
É importante ressaltar que a apresentação dos projetos ao CEP não visa constituir obstáculos para a realização da pesquisa, mas, ao contrário, estimulá-las sob a égide da ética e da proteção do ser humano. Segundo Prado $^{8}$, o trabalho desenvolvido no Brasil pelo Sistema CEP-CONEP, de inspiração bioética, tem se voltado para o debate permanente e de participação pluralista, com escopo de valorizar a dignidade do ser humano a partir do resgate dos valores morais e éticos da sociedade brasileira.

As instituições nas quais se realizem pesquisas envolvendo seres humanos devem constituir um ou mais de um CEP, conforme suas necessidades. Esse sistema iniciou-se em instituições de saúde. No entanto, como a participação de seres humanos em pesquisas abrange diferentes áreas de conhecimento, várias instituições de outras áreas participam desse processo de discussão como Direito, Sociologia, Educação e Antropologia.

A Resolução n. 466/125 define as características gerais dos comitês, sua composição e atribuições. Estabelece, ainda, que os CEP devam ser registrados na CONEP, que analisa a documentação enviada junto com a solicitação do registro, devendo conter: formulário com a relação dos membros e dados da instituição e do coordenador; ato de criação do comitê de ética pela instituição; breve descrição da missão e atividades gerais da instituição solicitante, incluindo as relativas às pesquisas; e documento de entidade da sociedade civil organizada, apresentando o representante de usuários, de acordo com a Resolução n. 240/979 . A CONEP avalia e envia documento aprovando o registro ou solicitando o atendimento de algum requisito definido na Resolução n. $370 / 07^{10}$.

Para Freitas ${ }^{7}$, a legitimidade do Sistema CEP-CONEP pode ser atribuída à grande aceitação da norma, seguramente reflexo da necessidade de criação de um sistema de acompanhamento de pesquisas no Brasil, já com resultados positivos para o funcionamento dos comitês como, por exemplo, a melhora da qualidade dos protocolos e a mobilização em torno da discussão ética.

\section{MÉTODO}

Trata-se de um estudo documental de caráter exploratório, descritivo e retrospectivo. Após autorização e concordância da CONEP, para utilização de documentos como, relatórios anuais e banco de dados, foi realizado, no período compreendido entre agosto e setembro de 2010, levantamento e análise dos registros do Sistema Gestão que opera internamente nas estruturas da instituição.

Para alcance dos objetivos propostos, realizou-se a apuração do quantitativo, bem como da área de formação dos membros que compõem os Comitês de Ética em Pesquisa existentes em todo o Brasil, cadastrados na CONEP.

As informações foram coletadas com a utilização de um sistema de consulta, cujos dados foram importados para outra base para classificação, para que, após essa etapa, se pudesse realizar uma checagem e uma filtragem dos registros encontrados no banco de dados.

A análise do Sistema Gestão contempla as atividades desenvolvidas pela CONEP entre 1996 e 2010, e permite o acesso às informaçôes, muitas delas necessárias para a realização deste estudo. Para o armazenamento dos dados foi utilizado o Excel e os dados foram dispostos em tabelas.

Quanto aos aspectos éticos, o presente trabalho, de abordagem quantitativa, teve como instrumento a base de dados da CONEP, da qual se extraiu as informaçôes necessárias, portanto não sendo exigida a apreciação de um CEP.

\section{RESULTADOS E DISCUSSÃO}

Os CEP são colegiados interdisciplinares e independentes, com múnus público e devem ser constituídos por um número não inferior a sete membros. Sua constituição deve incluir a participação de profissionais da área de saúde, das ciências exatas, sociais e humanas, incluindo, por exemplo, juristas, teólogos, sociólogos, filósofos, bioeticistas e, pelo menos um membro da sociedade representado os usuários da instituição. 
Artigo publicado no ano de 2003 por Freitas, et $\mathrm{al}^{11}$ revelou $384 \mathrm{CEP}$ cadastrados na CONEP, contabilizando 4.611 membros, e com uma média de 12 membros por comitê. O trabalho levantou a porcentagem referente às carreiras que compunham o Sistema CEP-CONEP, em junho de 2003. De acordo com os resultados, as profissões que predominavam nos CEP, em ordem decrescente, eram: Medicina, Ciências Humanas e Sociais, Enfermagem, Ciências Exatas, Advocacia, Ciências Biológicas, Farmácia-Bioquímica, Ciências da Saúde, Odontologia, Teologia-Filosofia, Serviço Social e Bioética ${ }^{11}$.

Observou-se que não havia uma delimitação clara das áreas de conhecimento e das profissóes representadas. Evidenciou-se este fato ao não se enquadrar em Ciências da Saúde áreas consideradas como pertencentes à mesma, como Medicina e Odontologia. O mesmo fato ocorreu com as demais áreas de conhecimento nas quais Direito e Serviço Social, por exemplo, estão separados das Ciências Humanas nos dados apresentados ${ }^{11}$.

Vale ressaltar que os CEP podem variar na sua composição, de acordo com seu regimento interno, dependendo das especificidades da instituição e das linhas de pesquisa a serem analisadas. Têm sempre caráter multi e transdisciplinar, não devendo haver mais que metade de seus membros pertencentes à mesma categoria profissional e devem contar com a participação de pessoas de ambos os sexos.

Kipper, et a ${ }^{12}$ afirmam que os membros de um comitê de ética em pesquisa revisam os estudos para decidir se são éticos. Para que se possa realizar esse trabalho, cada comitê precisa de uma combinação adequada de pessoas, sem que se perca, contudo, a noção de interdisciplinaridade.

Dessa forma, a composição e os procedimentos para tomada de decisão no CEP devem assegurar sua característica fundamental de independência relativa à influência política, institucional, hierárquica, corporativa, financeira e econômico-mercadológica.

Sobre a formação dos membros de um comitê de ética, os mesmos devem ter experiência em ciência e pesquisa. Os membros devem estar qualificados para revisar atividades específicas de pesquisa, bem como a aceitabilidade da pesquisa proposta em termos de compromissos e regulamentos institucionais, leis aplicáveis, e padrôes de conduta e prática profissional. Além disso, uma diversidade de gênero, faixa etária, e origens étnicas e culturais entre os membros de um comitê de ética devem existir para que uma revisão equilibrada da pesquisa possa acontecer.

O levantamento em questão contabilizou a existência de 9.075 membros atuantes em 2010 em 602 CEP registrados à época, com uma média de 16 membros por CEP, tendo representação das mais diversas áreas das Ciências da Saúde e também de outras áreas do conhecimento. No estudo realizado encontrou-se a representatividade em trinta e cinco diferentes áreas de conhecimento. A Medicina é a área de formação que apresenta maior número de membros atuantes em comitês.

A Tabela 1 mostra as trinta e cinco carreiras profissionais encontradas juntamente com o quantitativo de membros que compóem os Comitês de Ética em Pesquisa no ano de 2010. De acordo com a tabela, os dados tidos como não identificados (833), são aqueles cujo cadastro não foi possível identificar a qual área do conhecimento se enquadravam, pois constavam como: professor, aposentado, especialistas, secretária, agente da comunidade. Sabe-se que os membros existem, porém não se conseguiu identificar a sua respectiva profissão e, portanto, nota-se uma falha nos dados de $9,17 \%$ sobre a amostra. 
Revista B10ETHIKOS - Centro Universitário São Camilo - 2014;8(1):53-60

Tabela 1. Profissóes dos membros de CEP no ano de 2010

\begin{tabular}{|c|c|c|c|}
\hline Profissão & n. & $\%$ & Área de Conhecimento \\
\hline Medicina & 1870 & $20,61 \%$ & Ciências da Saúde \\
\hline Enfermagem & 1012 & $11,22 \%$ & Ciências da Saúde \\
\hline Direito & 481 & $5,31 \%$ & Ciências Sociais \\
\hline Ciências Biológicas & 479 & $5,27 \%$ & Ciências Biológicas \\
\hline Odontologia & 412 & $4,59 \%$ & Ciências da Saúde \\
\hline Fisioterapia & 411 & $4,52 \%$ & Ciências da Saúde \\
\hline Fonoaudiologia & 353 & $3,88 \%$ & Ciências da Saúde \\
\hline Filosofia e Teologia & 316 & $3,48 \%$ & Ciências Humanas \\
\hline Administraç̃ão & 175 & $1,92 \%$ & Ciências Sociais \\
\hline Educação Física & 122 & $1,34 \%$ & Ciências da Saúde \\
\hline Sociologia & 98 & $1,07 \%$ & Ciências humanas \\
\hline Matemática & 96 & $1,05 \%$ & Ciências Exatas \\
\hline Física & 91 & $1,01 \%$ & Ciências Exatas \\
\hline Química & 77 & $0,84 \%$ & Ciências Exatas \\
\hline Historia & 76 & $0,83 \%$ & Ciências Humanas \\
\hline Medicina Veterinária & 74 & $0,81 \%$ & Ciências Agrárias \\
\hline Probabilidade e Estatística & 41 & $0,45 \%$ & Ciências Exatas \\
\hline Agronomia & 35 & $0,38 \%$ & Ciências Agrárias \\
\hline Antropologia & 34 & $0,37 \%$ & Ciências Humanas \\
\hline Linguística & 21 & $0,23 \%$ & Linguística \\
\hline Zootecnia & 19 & $0,21 \%$ & Ciências Agrárias \\
\hline Bioética & 16 & $0,17 \%$ & \\
\hline Artes & 11 & $0,12 \%$ & Linguística \\
\hline Oceonagrafia & 04 & $0,04 \%$ & Ciências Exatas \\
\hline Dados não identificados & 833 & $9,17 \%$ & \\
\hline Total & 9075 & $100 \%$ & \\
\hline
\end{tabular}

Fonte: pesquisa dos autores, 2010. 
Conforme demonstrado, tanto em 2003 como em 2010 há um predomínio de membros na área de Ciências da Saúde representado, principalmente, por profissionais que atuam nas áreas de Medicina, Enfermagem, Odontologia. Pesquisas realizadas confirmam uma proporção maior de profissionais médicos atuando em Comitês de Ética em Pesquisa ${ }^{13,14,15,16}$.

No estudo realizado, encontra-se a tradicional liderança da área de saúde, constante na composição dos CEP. O número elevado desses profissionais demonstra a tendência em se acreditar que as pesquisas com seres humanos ocorrem quase que exclusivamente na área de Ciências da Saúde, no entanto, como afirmam Freitas e Novaes ${ }^{17}$, as diretrizes atualmente em vigor ampliam a abrangência de atuação para toda pesquisa envolvendo seres humanos e, portanto, sua aplicação ocorre não apenas na área da saúde, mas também nas pesquisas das diversas áreas do conhecimento que, seguramente, também necessitam de apreciação ética.

A Tabela 2 mostra as seis áreas de conhecimento juntamente com o seu quantitativo.

\begin{tabular}{lcc}
\multicolumn{3}{l}{ Tabela 2. Distribuição por área de conhecimento } \\
\hline Área de Conhecimento & $\mathbf{n .}$ & $\%$ \\
\hline Ciências da Saúde & 4844 & $54 \%$ \\
Ciências Humanas & 1098 & $13 \%$ \\
Ciências Sociais & 1008 & $11 \%$ \\
Ciências Biológicas & 479 & $5 \%$ \\
Ciências Exatas & 368 & $4 \%$ \\
Engenharias & 215 & $2 \%$ \\
Ciências Agrárias & 128 & $1 \%$ \\
Linguística & 102 & $1 \%$ \\
Outros & 833 & $9 \%$ \\
Total & 9075 & $100 \%$ \\
\hline
\end{tabular}

Fonte: pesquisa dos autores, 2010.

A área de Ciências da Saúde concentra 54\% de membros, daí decorre a grande existência de CEP vinculados a essa área. Esses CEP existem, principalmente, em centros de pesquisa biológicas, hospitais públicos ou particulares, hospitais-escolas de universidades e nas faculdades de Medicina, Enfermagem, Odontologia, Farmácia, locais tradicionalmente associados a pesquisas biomédicas e também abertos às demais áreas de conhecimento para que apresentem seus projetos.
Observa-se, a partir da Tabela 1, que a forma de catalogação dos dados passa a contemplar outras profissões sendo que os dados não se encontram distribuídos de forma conjunta abarcando profissōes e áreas de conhecimento. Os dados de 2010 já permitem avaliar o quantitativo de psicólogos inseridos em comitês de ética, pois em $2003^{18}$ não havia como afirmar se esses profissionais estavam inseridos ou não nos dados referentes ao quantitativo de Ciências Humanas. O mesmo ocorre com outras áreas como Fisioterapia e Fonoaudiologia, pois anteriormente também não era possível quantificar e afirmar se esses profissionais estavam ou não representados na área de Ciências da Saúde.

Pode-se observar que, embora exista uma grande adesão de profissionais da área da saúde historicamente envolvidos com a questão, paulatinamente profissionais de outras áreas, como Educação, Direito, Sociologia, Antropologia, têm aderido ao processo de avaliação ética, reconhecendo a importância e a legitimidade do Sistema CEP-CONEP.

$\mathrm{O}$ aumento do quantitativo de membros de CEP nos últimos anos reflete o crescimento e o interesse não somente pela produção de pesquisas biomédicas e pelos avanços da biotecnociência, mas também a preocupação com os aspectos éticos dessa produção. O despertar dessa preocupação como afirma Barbosa ${ }^{19}$ "ressalta a missão educativa e de formação de uma consciência ética e de responsabilidade". Ainda de acordo com Barbosa ${ }^{19}$, a bioética é hoje "um dos campos do conhecimento acadêmico que mais cresce nos últimos anos".

Nesse sentido, profissionais como, por exemplo, os fisioterapeutas vêm despertando o interesse crescente por temas ligados à Bioética, pois como afirma Badaró e Guilhem $^{20}$, é importante que a Fisioterapia esteja inserida nesse contexto de discussão que poderá contribuir para identificar conflitos ligados à prática clínica.

É oportuno ressaltar que, paralelamente ao crescimento no número de membros atuantes nos CEP, houve um incremento na quantidade de comitês registrados no Brasil. É possível inferir que uma cultura Bioética, e, sobretudo, em ética em pesquisa com seres humanos, vem se fortalecendo ano a ano, ratificando o esforço e o empenho da CONEP para a condução das pesquisas de forma ética e segura. 


\section{CONCLUSÃO}

Com o funcionamento regular dos CEP, infere-se o aumento ano a ano dos projetos captados pelo Sistema, o que traduz a estruturação progressiva do Sistema CEP-CONEP e um incremento real das atividades de pesquisa no País. Observou-se, também, uma proporção cada vez maior na interdisciplinaridade dos membros que compõem esses comitês, por meio da adesão de áreas anteriormente pouco presentes.

No período analisado, pode-se perceber o estabelecimento de uma nova mentalidade com relação às pesquisas com seres humanos no país, verificado tanto pela ampla adesão na implantação de um sistema descentralizado de acompanhamento de projetos, com mais de 600 CEPs cadastrados, quanto pelos resultados práticos e concretos da ação desses comitês. A eficácia do
Sistema também mostrou interdisciplinaridade entre os membros dos comitês de ética no Brasil que compõem o CEP, com diversidades de profissões.

Os dados mostram a tradicional hegemonia de profissionais que pertencem à área de Ciências da Saúde, com predomínio significativo da Medicina, comprovado desde os primeiros levantamentos divulgados, além de Enfermagem, Odontologia e Farmácia. Observa-se, no entanto, um crescimento considerável de categorias como a Fisioterapia, a Fonoaudiologia e a Psicologia, o que permite inferir o interesse e o alcance que a ética em pesquisa tem despertado nessas áreas.

Por fim, o controle social desempenhado pelos CEPs está diretamente relacionado à eficiência na atuação de seus membros, cujo caráter de voluntariado lhes confere autonomia para desempenhar suas funções sem conflitos de interesse.

\section{REFERÊNCIAS}

1. Werner JA, Velho MTC. O Sistema CEP/CONEP no Brasil: discurso e realidade dez anos depois. Rev Direito UNISC. 2008;29:166-80. 2. Freitas CBD, Novaes HMD. Lideranças de comitês de ética em pesquisa no Brasil: perfil e atuação. Bioét. 2010;18(1):185-200.

3. Schramm FR. Aspectos cognitivos, metodológicos e morais do trabalho dos CEP. In: Carneiro F, organizador. A moralidade dos atos científicos. Rio de Janeiro: Fiocruz; 1999. p. 53-9.

4. Francisconi CFM, Goldim JR. Ética aplicada à pesquisa. Cadernos Ética Pesquisa. 2002;V(9):8-9.

5. Conselho Nacional de Saúde. Resolução n. 466, de 12 de dezembro de 2012. Diário Oficial da União n. 12, em 13 de junho de 2013, Seção 1, p. 59.

6. Massarollo MCKB, Kurcgant P, Fernandes MFP. Comitê de Ética em Pesquisa da Escola de Enfermagem da USP: experiência dos primeiros seis anos. Rev Esc Enferm USP. 2009;43(Esp 2):1303-7.

7. Freitas CBD. A legitimidade e os valores éticos expressos na Resolução n. CNS 196/96. In: Carneiro F, organizador. A moralidade dos atos científicos. Rio de Janeiro: Fiocruz; 1999. p. 73-7.

8. Prado MM. Panorama da bioética no Brasil: um estudo sobre a reflexão bioética desenvolvida no país [dissertação]. Brasília: Universidade de Brasília; 2002. 203 p.

9. Conselho Nacional de Saúde. Resolução n. 240/97 de 19 de março 1997. Dispõe sobre Normas de Pesquisa em Saúde. Brasília: Ministério da Saúde; 1997.

10. Conselho Nacional de Saúde. Resolução n. 370/07 de 24 de julho 1998. Dispõe sobre Normas de Pesquisa em Saúde. Brasília: Ministério da Saúde; 1997.

11. Freitas CBD, Lobo MO, Gonçalves GB. A atuação da Conep. Cadernos Ética Pesquisa. 2003;VI(12):5-11.

12. Kipper D, Loch JA, Ferrari NM. A experiência do comitê de ética em pesquisa da Pontifícia Universidade Católica do Rio Grande do Sul, do comitê de bioética do Hospital São Lucas e da Faculdade de Medicina da PUCRS. Bioét. 1998;6(2):203-9.

13. Campbell EG, Weissman JS, Clarridge B, Yucel R, Causino N, Blumenthal D. Characteristics of medical school faculty members serving on institutional review boards: results of a national survey. Acad Med. 2003;78(8):831-6.

14. Valdez-Martinez E, Trumbull B, Garduño-Espinosa J, Porter JD. Understanding the structure and practices of research ethics committees through research and audit: a study from Mexico. Health Policy. 2005;74(1):56-68.

15. Hardy E, Bento SF, Osis MJD, Hebling EM. Comitês de ética em pesquisa: adequação à Resolução n. 196/96. Rev Assoc Med Bras. 2004;50(4):457-62. 
16. Goldim JR, Francisconi CF, Matte U, Raymundo MM. A experiência dos comitês de ética em pesquisa no Hospital das Clínicas de Porto Alegre. Bioét. 1998;6(2):211-6.

17. Freitas CBD, Novaes HMD. Posicionamento de lideranças do sistema de avaliação da ética em pesquisa no Brasil - consensos e divergências. Bioét. 2007;15(1):101-16.

18. Freitas CBD, Lobo M, Hossne WS. Oito anos de evolução: um balanço do sistema CEP-CONEP. Cadernos Ética Pesquisa. 2005; VI(16):20-30.

19. Barbosa S. Bioética no Estado Brasileiro: situação atual e perspectivas futuras. Brasília: Ed. UnB; 2010. 188 p.

20. Badaró AFV, Guilhem D. Bioética e pesquisa na Fisioterapia: aproximação e vínculos. Fisioter Pesq. 2008;15(4):402-7. 